2005

The correlation grid: analysis of synchronous spiking in multi-dimensional spike train data and identification of feasible connection architectures

Stuart, EJ::0000-0001-8373-8526

http://hdl.handle.net/10026.1/8545

10.1016/j.biosystems.2004.09.011

BIOSYSTEMS

All content in PEARL is protected by copyright law. Author manuscripts are made available in accordance with publisher policies. Please cite only the published version using the details provided on the item record or document. In the absence of an open licence (e.g. Creative Commons), permissions for further reuse of content should be sought from the publisher or author. 


\title{
The Correlation Grid: Analysis of Synchronous Spiking in Multi-dimensional Spike Train Data and Identification of Feasible Connection Architectures
}

\author{
L. Stuart ${ }^{*}, M$. Walter ${ }^{*}$ and R. Borisyuk ${ }^{\dagger \ddagger}$ \\ *The Visualization Lab, Centre for Intelligent Interactive Systems, University of Plymouth, Plymouth, UK \\ ${ }^{\dagger}$ Centre for Theoretical and Computational Neuroscience, University of Plymouth, Plymouth, UK \\ ${ }^{\ddagger}$ Institute of Mathematical Problems in Biology, Russian Academy of Sciences, Pushchino, Moscow Region 142 290, Russia
}

\begin{abstract}
This paper presents a visualization technique specifically designed to support the analysis of synchronous firings in multiple, simultaneously recorded, spike trains. This technique, called the Correlation Grid, enables investigators to identify groups of spike trains, where each pair of spike trains has a high probability of generating spikes approximately simultaneously or within a constant time shift. Moreover, the correlation grid was developed to help solve the following reverse problem: identification of the connection architecture between spike train generating units, which may produce a spike train dataset similar to the one under analysis. To demonstrate the efficacy of this approach, results are presented from a study of three simulated, noisy, spike train datasets. The parameters of the simulated neurons were chosen to reflect the typical characteristics of cortical pyramidal neurons. The schemes of neuronal connections were not known to the analysts. Nevertheless, the correlation grid enabled the analysts to find the correct connection architecture for each of these three data sets.
\end{abstract}

\section{Introduction}

Synchronisation of neural discharges is considered to be an important principle of information processing by cortical neural circuits (Nase et al. 2003; Neuenschwander et al., 2003, Schmidt 2003). Analysis of synchronisation of simultaneously recorded spike trains is usually based on the calculation of a counting function, such as a crosscorrelation function or a cross-correlogram (Gerstein and Kirkland, 2001). Cross-correlograms are a common and useful means of representing the relationship between pairs of spike trains, recorded in this way.

However, for any significantly sized neuronal architecture, numerous cross-correlograms would require in-depth analysis in order to identify synchronous activity in and between groups of spike trains. Indeed, investigation into the functional connectivity of neuron groups is a very important area of research.

The objective is the identification of a feasible architecture of connections between elements that could account for the original spike train data and subsequent correlation functions. Typically in mathematics, the reverse problem is very difficult to solve and non-unique solutions may exist. Nevertheless, the results of our blind testing are promising.

Furthermore, new problems are posed by the increasingly large neural assemblies that are currently recorded.

In this paper, the role that information visualization may play in alleviating some of these problems is discussed. A visualization technique, called the Correlation Grid (Walter et al. 2003), is used to analyse a simultaneously recorded dataset of $n$ spike trains. Subsequently, this grid is used to identify clusters of synchronous spike trains. Thus, it supports the proposal of a scheme of functional connectivity based on the fact that high correlation between spike trains corresponds to significant functional connection.

In order to imitate the experimental data of multispike train recordings, a biologically-inspired generator of spike trains with interconnections according to a predefined connection scheme, (Borisyuk 2002) is used. Each spike train is generated on the basis of an enhanced integrate and fire model and some specified connection architecture with particular synaptic weights. Note that all results in this paper are obtained in the regime of blind testing. Only the spike trains were made available for analysis. These spike trains are used to generate the Correlation Grid, which is analysed and used to identify the underlying connection architecture of neurons. Finally, this proposed architecture is assessed with respect to the original used to generate the data.

Progressively, the number of correct connection identifications has increased during empirical testing, resulting in improvements to the methodology used. Currently, it is possible to obtain a completely accurate connection scheme. In this paper, three trials are presented, which correspond to a different number of spike trains and/or connection schemes. 


\section{Brillinger normalisation of cross- correlogram}

The cross-correlogram for each pair of spike trains is calculated in order to create the correlation grid. To create the cross-correlogram of a 'target' spike train relative to a 'reference' spike train, a standard counting function (Gerstein 2001, MuLab) is used. This function counts the number of spikes in the reference spike train relative to the spikes in the target spike train. Figure 2-1(i) depicts a typical cross-correlogram for a pair of connected neurons with a time shift corresponding to the delay in spike propagation from one neuron to another. In contrast, a typically flat cross-correlogram for a pair of unconnected neurons is shown in Figure 2-1(ii).

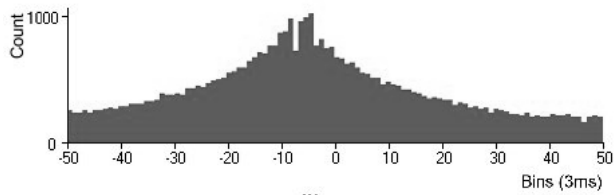

(i)

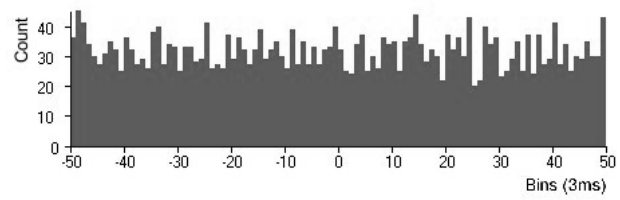

(ii)

Figure 2-1 An example of a cross-correlogram of (i) two correlated spike trains and (ii) two uncorrelated spike trains

In order to make statistically significant judgements regarding the nature of the peaks, in the crosscorrelogram, the Brillinger normalisation is applied to the data (Brillinger 1979) and the peaks exceeding the higher bound of the $95 \%$ confidence interval are considered to be significant peaks. Figure 2-2 shows a normalised cross-correlograms for the same data as shown in Figure 2-1 and boundaries of $95 \%$ confidence interval. The highest significant peak is referred to as the 'main' peak. Thus, the main peak can be considered to be a measure of the proximity of two spike trains.

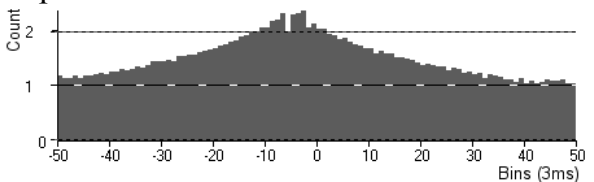

(i)

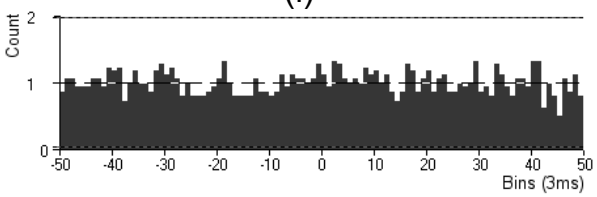

(ii)

Figure 2-2 An example of a Brillinger normalised cross correlogram with confidence interval of (i) two correlated spike trains and (ii) two uncorrelated spike trains.
Subsequently, the value of this main peak is used to quantify the distance between pairs of spike trains in the clustering algorithm as well as the density of shading in the corresponding cell of the correlation grid.

\section{The correlation grid}

The correlation grid is an overview of multiple cross-correlograms for a number of spike trains. Hence, for a given dataset, of $n$ spike trains, all pair wise cross-correlograms are generated and normalised using the Brillinger method, and the main peaks $c_{i, j},(i=1, \ldots, n, j=1, \ldots, n)$ are calculated for all pairs. Finally, the results are displayed as an $n$-by- $n$ symmetrical grid of grey scale cells, representing correlations between all pairs of spike trains. Thus, the magnitudes of main peaks are encoded from white, representing a nonsignificant peak, to black, representing the largest peak in the grid. The user has the flexibility to view 'all peaks' or solely significant peaks. Significant peaks are those that exceed the higher bound of the confidence interval. In this paper, we analyse neural circuits with positive connection strengths only. When the main peak of the cross-correlogram exceeds the higher bound of the Brillinger interval, a positive functional connection exists between the neurons generating these spike trains. In principle, negative connections should also be considered as they will result in peaks that are lower than the low bound of the significance interval.

Additionally, it is useful to reorder the rows and columns of correlation grid in order to highlight the inherent relationships between multiple spike trains. The method used to accomplish this reordering is the cluster analysis algorithm.

\subsection{The clustering algorithm}

Let us consider an $n$-by- $n$ correlation grid of cells as a matrix of similarities between objects (spike trains). Thus, the pair of spike trains who crosscorrelogram has the largest main peak is considered to be the most similar (the most correlated) pair of spike trains.

In the trials, different algorithms were used to cluster spike trains. These included the following methods: nearest neighbour (the minimum of measures between objects in two groups), furthest neighbour (the maximum of measures between objects in two groups), and a centroid clustering algorithm. In conclusion, the most efficient algorithm is the furthest neighbour method. Intuitively this algorithm creates tight clusters and all objects inside the cluster have limited dissimilarity. 


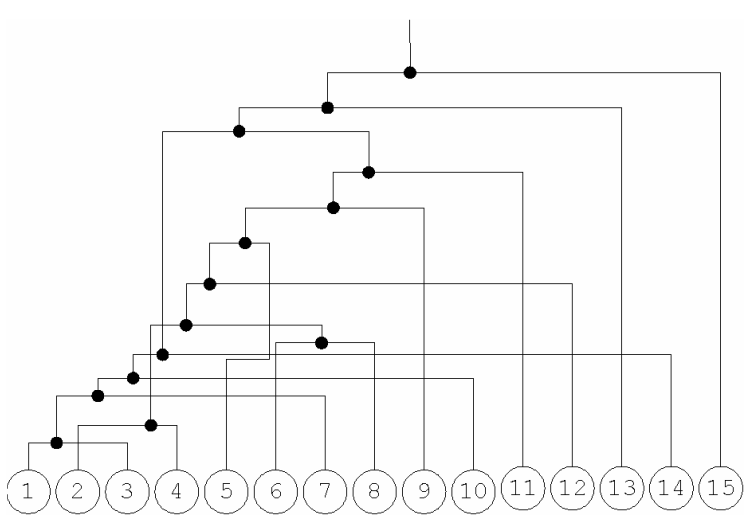

Figure 3-1 Cluster analysis dendrogram for trial one data, also shown as the correlation grid in Figure 4-3.

Figure 3-1 shows a cluster analysis dendrogram for the data from trial one. This data is also depicted as the 'initial' correlation grid shown in Figure 4-3. In order to enhance the perception of groups within this correlation grid, a left-hand recursive algorithm is used to redefine the ordering of spike trains.

This algorithm descends this binary tree (see the dendrogram, Figure 3-1) and at each node, it initially follows the leftmost branch. Thus, the algorithm recursively follows the leftmost branch until it reaches a leaf node. Upon finding a left node, the algorithm then traverses the rightmost branch of the current sub-tree before ascending back up the tree to find the next rightmost branch to be followed.

Subsequently, this new ordering is used to re-order the spike trains in the correlation grid. The resultant correlation grid is shown in Figure 4-5. This shows a significant improvement to the visualization of these groups of synchronously spiking trains. From this grid, it is immediately possible to infer that all spike trains are arranged into two separate clusters: $(1,3$, 7, 10 and 14) and (2, 4, 6, 8, 12, 5, 9, and 11) and also that spike trains 13 and 15 are independent.

Additionally, it is immediately notable that the second main cluster has some overlapping connections that require further analysis.

\section{Data analysis and connection structure identification}

In this section, the results of three trials are presented. Each dataset was generated using simulations of integrate and fire neurons with particular coupling between elements. The parameters of the model were chosen to mimic the general neurophysiological characteristics of cortical neurons. All connection strengths in these simulations were chosen to be positive.

For example, in trial one, an assembly of 15 neurons was simulated for a period of $20000 \mathrm{~ms}$. The mean inter-spike interval (ISI) was $75 \mathrm{~ms}$, the standard deviation of the ISI of the dataset was $53 \mathrm{~ms}$ and its coefficient of variation was 0.7 . The ISI Histogram for spike train number 6 is shown in Figure 4-1 (a) and its autocorrelation is shown in Figure 4-1. This figure depicts (a) the ISI histogram of spike train 6 and (b) the autocorrelation of spike train 6 from the trial one dataset.

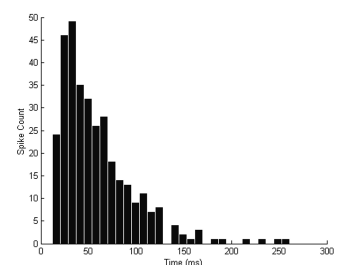

(a)

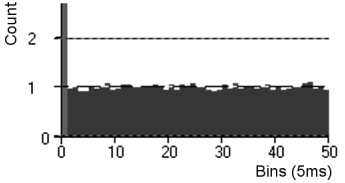

(b)
Figure 4-1 This figure depicts (a) the ISI histogram of spike train 6 and (b) the autocorrelation of spike train 6 from the trial one dataset.

The raster plot of the first $3000 \mathrm{~ms}$ of this data is also shown in Figure 4-2.

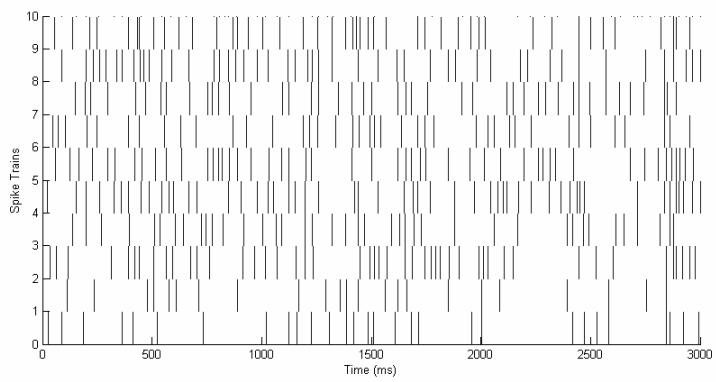

Figure 4-2 Raster plot of trial one spike trains

We call this analysis "blind trials" because the only spike trains have been available for analysis.

\subsection{Trial One. Creating the correlation grid}

Initially, the cross-correlogram for each pair of fifteen spike trains is calculated with a bin size of $1 \mathrm{~ms}$ and a time-window of $100 \mathrm{~ms}$ (100 bins). The correlation grid for the first data set is shown in Figure 4-3.

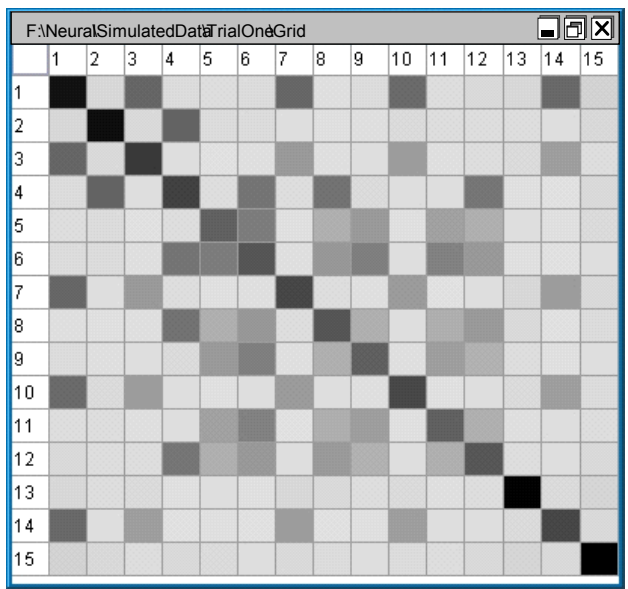

Figure 4-3 The initial correlation grid for trial one data

For step two, all peaks that do not exceed the higher bound of the confidence interval of the Brillinger normalization are ignored. Thus, Figure 4-4 solely 
shows the significant values of the main peaks as insignificant peaks are represented in white.

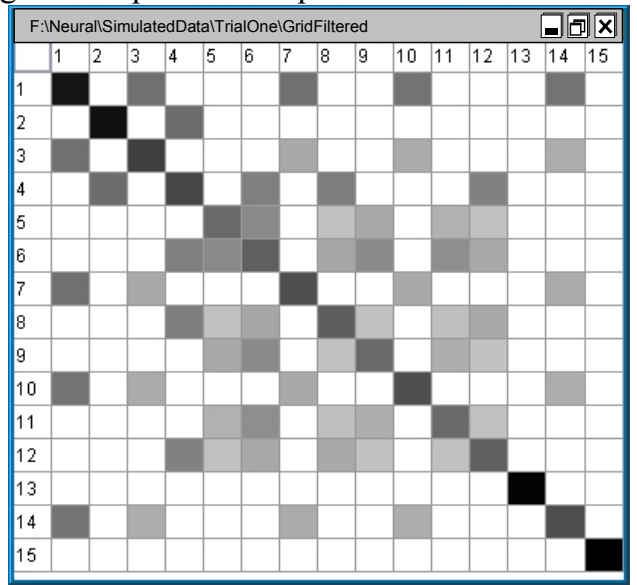

Figure 4-4 The filtered correlation grid for trial one data

The final stage of creating the correlation grid is the application of the clustering algorithm. Figure 4-5 shows the striking affect that clustering may have on a correlation grid. This improved ordering supports the identification of groups and their position within any hierarchies that exist. The interpretation of the correlation grid is discussed in the following section.

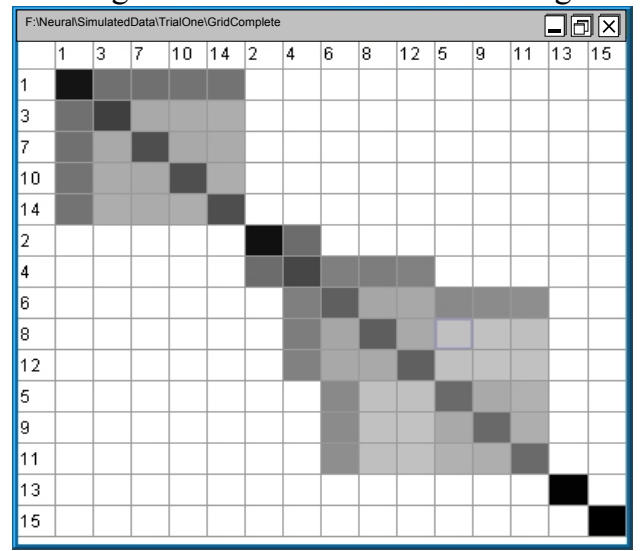

Figure 4-5 The filtered and reordered correlation grid for trial one dato

\subsubsection{Interpretation of the correlation grid}

Initial inspection of the grid reveals that three main groups exist. Let us refer to these as the upper, middle and lower groups. These groups are indicated by dashed boxes in Figure 4-6.

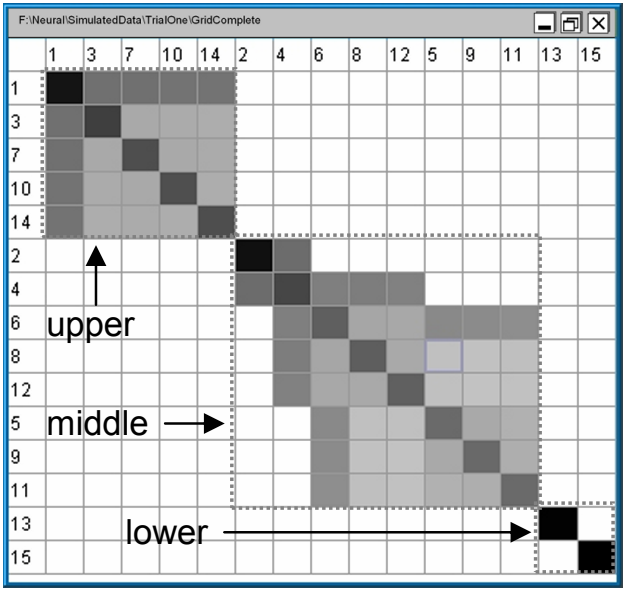

Figure 4-6 The final correlation grid depicting the three main groups in the trial one dataset

The top and middle groups are examined in significant detail in sections 4.1.3 and 4.1.4.

The lower group is very clear. As there is no indication of any significant relationships of either neuron with any other neuron in the assembly, it is feasible to conclude that both neurons 13 and 15 are completely unconnected.

\subsubsection{The upper group of trial one}

In order to interpret the upper group, the top portion of the correlation grid is enlarged and is shown in Figure 4-7.

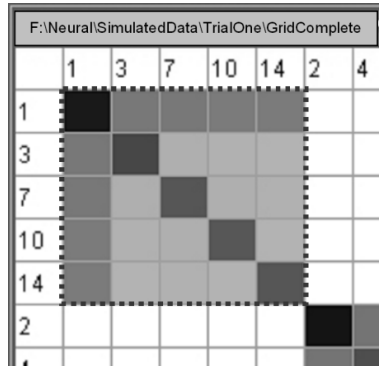

Figure 4-7 Enlargement of the upper group of the correlation grid of trial one data

Note that the upper group is made up of neurons 1, $3,7,10$ and 14. Further, there is a stronger correlation (denoted by the darker grey shade) between neuron 1 and all of the other neurons in the group. Note that neurons 3, 7, 10 and 14 exhibit a correlation to one another. Thus, it is likely that neuron 1 is connected to all of the other neurons: 3 , 7,10 and 14 . Thus, it is likely that the correlation between neurons $3,7,10$ and 14 is due to the fact that they have a common input.

This hypothesis is confirmed by closer inspection of the cross-correlograms of the neuron pairs $(1,3)$ and $(3,7)$, shown in Figure 4-8 and Figure 4-9, respectively. 


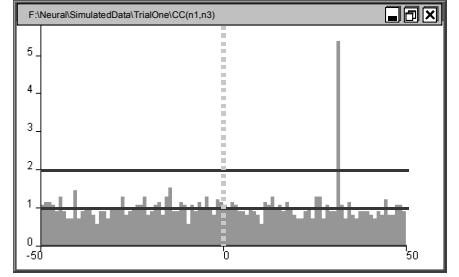

Figure 4-8 The cross-correlogram of spike trains 1 and 3 of trial one

Figure 4-8 depicts the correlation of neurons 1 and 3. As anticipated, there is a time delay between the spikes of these neurons. From the diagram, it is possible to deduce that there is a high probability that a spike will be generated by neuron 3 approximately $25-30 \mathrm{~ms}$ after neuron 1 spikes. Thus, there is an excitatory connection from neuron 1 to neuron 3 .

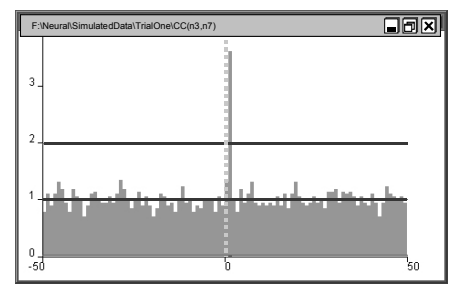

Figure 4-9 The cross-correlogram of spike trains 3 and 7 of trail one

Figure 4-9 depicts the correlation of neurons 3 and 7. As anticipated, there is direct synchronous spiking activity between these two neurons due to the fact that both are stimulated by neuron 1 .

All of these observations support the hypothesis that the sub-assembly of the upper group is a circuit with a common source. This is depicted in Figure 4-10.

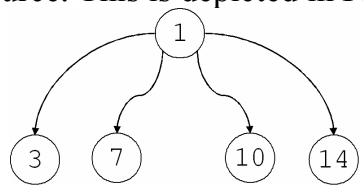

Figure 4-10 The neuronal assembly of the upper group of trial one

\subsubsection{The middle group of trial one}

In order to interpret the middle group, the middle portion of the correlation grid is enlarged and shown in Figure 4-11.

From this Figure, three main groups are apparent. The 2-group of neurons 2 and 4, the 4-group made up of neurons 4, 6, 8 and 12 and the 6-group made up of $6,8,12,5,9$ and 11 . Note that the 2-group and 4-group overlap, as do the 4-group and 6-group. This overlapping indicates that there are connections between these groups and the overlapping neurons in principle could provide these couplings.

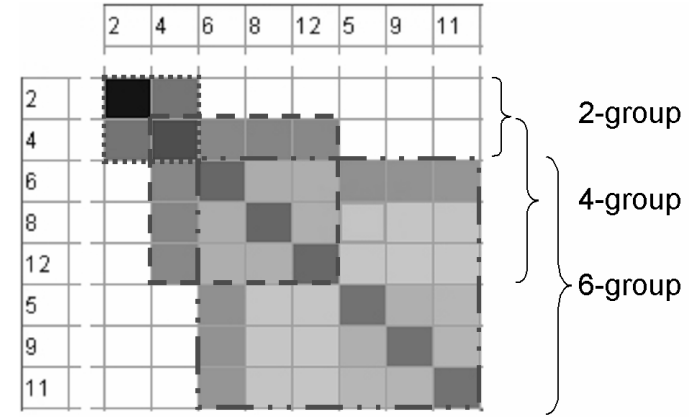

Figure 4-11 Enlargement of the middle group of the correlation grid of trial one data

The 2-group is relatively straightforward. It depicts neuron 2's influence on the spike trains of neuron 4. The 4-group has a similar structure depicted in Figure 4-7. Thus, it is possible to infer that neuron 4 is a common input to neurons 6,8 and 12 .

The 6-group requires further analysis. Thus, it is shown again in Figure 4-12. In this figure, the different values of the main peaks within the 6group are highlighted using solid lines. Very high correlation exists between neuron 6 and neurons 5, 9 and 11. High correlation exists between these neurons 5, 9 and 11. Finally, there is a low correlation between neurons 8 and 12 with neurons 5,9 and 11 .

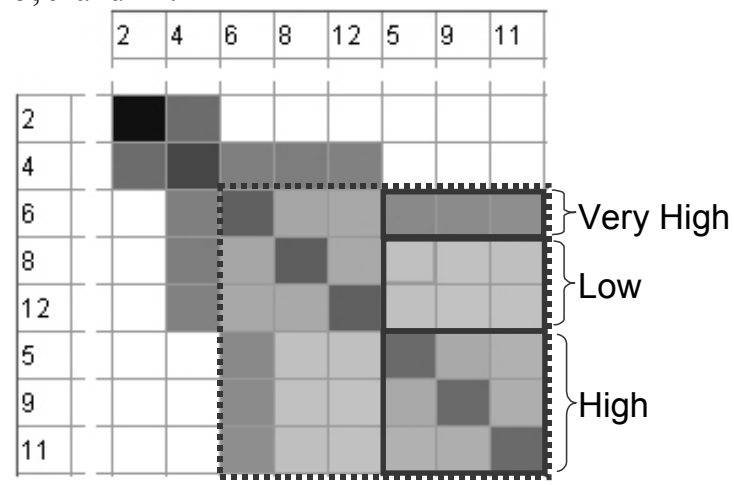

Figure 4-12 Enlargement of the middle group of the correlation grid of trial one data with further classification

The higher correlations form a familiar hierarchical pattern. Thus, neuron 6 connects to 5,9 and 11 . However, this is still some ambiguity regarding the connections between neurons 5, 9 and 11 with neurons 8 and 12 .

It is probable that this low correlation is attributable to the fact that neuron 4 is a common input to neurons 8 and 12 and also to neurons 5, 9 and 11 via neuron 6. To verify this assertion, the crosscorrelogram of neurons 8 and 11 , shown in $4-13$, is examined. As anticipated, there is a delayed correlation due to the fact that neuron 4 excites both neurons 8 and 11; however the excitation of neuron 11 is via neuron 6 accounting for the delayed correlation peak in Figure 4-13. 


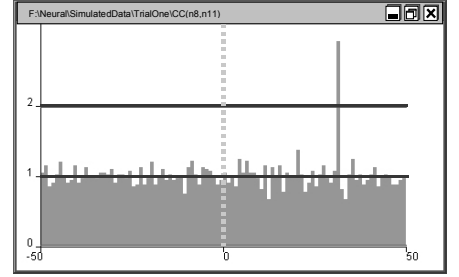

Figure 4-13 The cross-correlogram of spike trains 8 and 11 of trial one

\subsubsection{Summary of trial one observations}

From this analysis, it is now possible to re-create the coupling structure of this assembly of neurons based on the correlation grid and details from some of the cross-correlograms. This is depicted in Figure 4-14. After completion of the analysis we have compared the result obtained with connection scheme used in simulations and have found their complete correspondence.

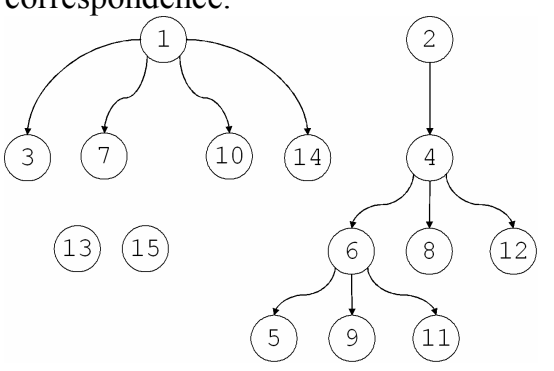

Figure 4-14 The neuronal assembly for trial one

\subsection{Trial two}

For this trial, an assembly of 10 neurons were simulated for a period of $100000 \mathrm{~ms}$. The raster plot for a portion of this data is shown in Figure 4-15. Note that the architecture of connections of the neurons in this assembly was unknown to the analysts prior to the investigation. Furthermore, the results of the analysis yielded an entirely accurate neural architecture.

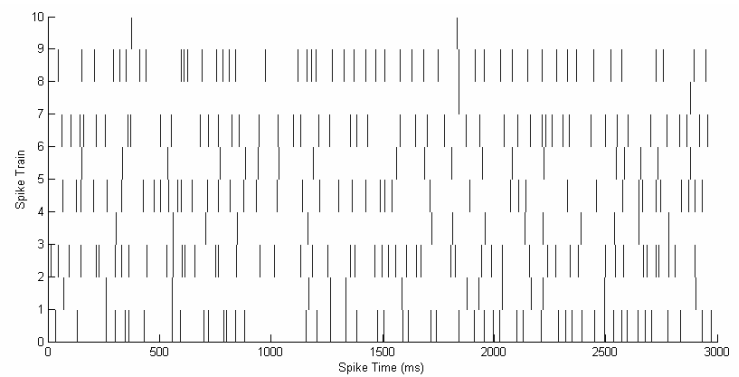

Figure 4-15 Raster plot of trial two spike trains

\subsubsection{The correlation grid for trial two}

Initially, the correlation grid was generated for this data, based on calculations of cross-correlograms with a bin size of $1 \mathrm{~ms}$ and a time-window of $100 \mathrm{~ms}$ (100 bins). Subsequently, the grid was filtered and clustered according to algorithms described above and the resultant correlation grid is shown in Figure 4-16.
Initial inspection of the grid reveals that there is considerable interconnection within this data set. In particular, note the distribution of grey cells in the grid. This grid can be partitioned into three groups: the upper, middle and lower groups as depicted in Figure 4-16. The upper and lower groups of trial two are discussed in detail in sections 4.2.2 and 4.2.3 respectively.

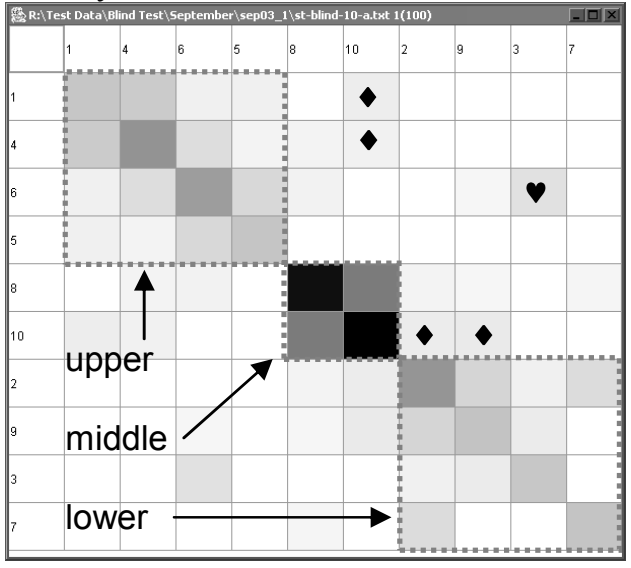

Figure 4-16 The final correlation grid depicting the three main groups of the trial two dataset

The most dominant feature of the correlation grid shown in Figure 4-16 is the correlation between spike trains 8 and 10, the middle group as indicated in the diagram. Generally, this feature infers a direct relationship between the two neurons. Further examination of the cross-correlogram of this pair (time delay in spike propagation from neuron 10 to 8 ), confirms that there is a connection from neuron 10 to neuron 8 .

Another dominant feature of the correlation grid shown in Figure 4-16 is that all three groups are somehow linked to neuron 10 . This is confirmed by the shading in all of the cells labelled with a diamond $(\bullet)$. This indicates that neuron 10 is likely to be the root of the assembly.

\subsubsection{The upper group of trial two}

In order to interpret the upper group of trial two, the top portion of the correlation grid is enlarged and shown in Figure 4-17.

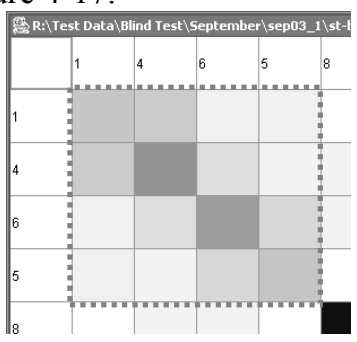

Figure 4-17 Enlargement of the upper group of the correlation grid of trial two data

Note that the upper group consists of neurons 1, 4, 6, and 5. Further, there is a stronger correlation between spike trains 1 and 4; 4 and 6; 6 and 5 in comparison to the remainder. Therefore, it is likely 
that these pairs are connected. Examination of their respective cross-correlograms confirms this hypothesis.

The correlation between spike trains 1 and 6 is significant but unclear. Therefore, the crosscorrelogram for this pair, shown in Figure 4-18, is examined. Note that in this cross-correlogram the main peak occurs around $15 \mathrm{~ms}$. Thus, there is a time delay in the spike propagation from neuron 1 to neuron 6. There is also a second (less significant) peak with a slightly larger time delay.

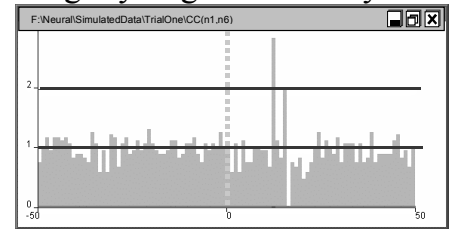

Figure 4-18 The cross-correlogram of spike trains 1 and 6 of trial two

These time delays indicate that the corresponding neurons are connected via some intermediate neuron(s). The presence of two peaks in the crosscorrelogram suggests that two different paths exist between the neurons 1 and 6 .

The strength of correlation between neurons 4 and 5 also indicates significant correlation. Upon closer inspection, the cross-correlogram of spike trains 4 and 5 shows a correlation peak close to zero (see Figure 4-19). This indicates that these neurons spike synchronously. Thus, suggesting that the two neurons are receiving common input.

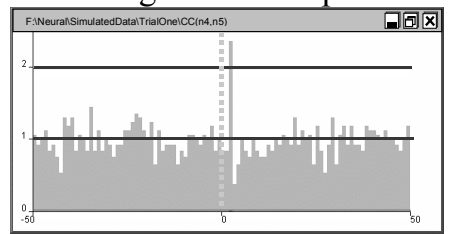

Figure 4-19 The cross-correlogram of spike trains 4 and 5 of trial two

From these observations, it is possible to deduce the connection scheme shown in Figure 4-20. This architecture shows that neuron 1 is a common source to neurons 4 and 5 and that they both have connections to neuron 6 .

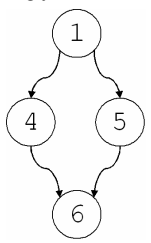

Figure 4-20 The neuronal assembly of the upper group of trial two

\subsubsection{The lower group of trial two}

In order to interpret the lower group, the lower portion of the correlation grid is enlarged and shown in Figure 4-21.

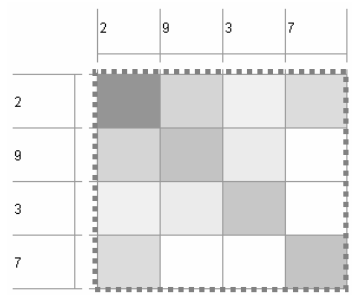

Figure 4-21 Enlargement of the lower group of the correlation grid of trial two data

From this figure, it is apparent that spike train 2 correlates strongly with both spike trains 7 and 9 . Likewise, spike train 9 correlates with spike train 3 . In addition, spike train 2 correlates, less strongly, with spike train 3 . It is possible to hypothesise from these observations that a connection exists between neuron 9 and both neurons 2 and 3. Similarly, that there is a connection between neurons 7 and 3 . Inspection of the cross-correlograms for these pairs confirms this hypothesis.

It is also possible to specify the direction of these connections, as shown in Figure 4-22. This structure also explains the weaker correlation between spike trains 2 and 3, as these neurons both receive input from neuron 9 .

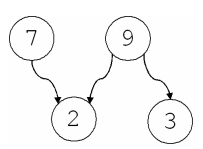

Figure 4-22 The neuronal assembly of the lower group of trial two

\subsubsection{Interconnection of upper and lower groups in trial two}

As hypothesised previously, neuron 10 links both the upper and lower groups. By investigating the cross-correlograms of spike train 10 with each of the upper and the lower groups (not shown here due to space limitations), it is possible to deduce that neuron 10 connects to both neurons 9 and 1 .

From the grid, it is also possible to observe another link, between the upper and lower groups. This correlation is between spike trains 3 and 6 and is indicated by the heart symbol $(\vee)$ in Figure 4-16.

Note that the cross-correlogram of neurons 3 and 6 showed a single peak, slightly delayed, thus indicating a direct connection between neurons 3 and 6.

\subsubsection{Summary of trial two observations}

From this analysis, the overview and details of the correlation grid, it is possible to deduce the underlying neuronal assembly of the data set, as depicted in Figure 4-23. The structure of this assembly also explains the weak correlations, shown in the grid, which have not yet been considered. 


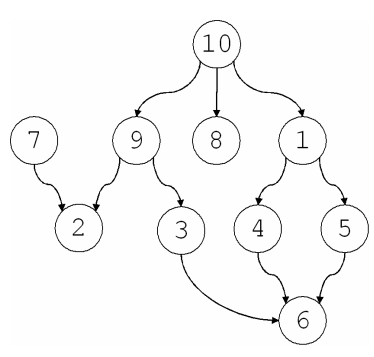

Figure 4-23 The neuronal assembly of trial two

The correlation between spike trains 10 and 4 is clear; neuron 10 is connected to neuron 4 via neuron 1. Likewise, the correlation between spike trains 8 and 9 is due to common input. The correlations between spike trains 4 and 8; 6 and $8 ; 2$ and 8 is not immediately obvious. However, these correlations are attributable to the fact that all inputs are governed by neuron 10 .

This assembly was completely and correctly reassembled based on the data available from the cross-correlograms and the correlation grid.

\subsection{Trial three}

For this trial, an assembly of 10 neurons were simulated for a period of $300000 \mathrm{~ms}$. Note that the architecture of connections of the neurons in this assembly was unknown to the analysts prior to the investigation. Again, the results of the analysis yielded an entirely accurate neural architecture.

\subsubsection{The correlation grid for trial three}

A correlation grid was generated for this data, with a bin size of $1 \mathrm{~ms}$ and a time window of 100 bins. The grid was subsequently filtered and clustered; the resultant correlation grid is shown in Figure 4-24.

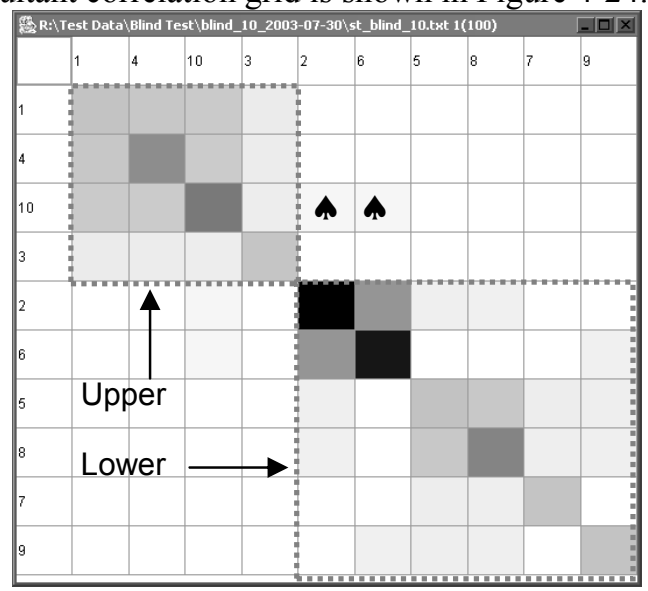

Figure 4-24 The final correlation grid depicting the two main groups of the trial three dataset

Initial inspection of the grid reveals that two main groups exist. Let us refer to these as the upper and lower groups as indicated in Figure 4-24. These groups are examined in detail in sections 4.3 .2 and 4.3.3.

In addition, there appears to be a link between the upper and lower groups. Note the correlations between spike trains 2 and 10 and spike trains 6 and 10 , indicated by the spade symbols ( $\mathbf{\uparrow})$ in Figure 4-24.

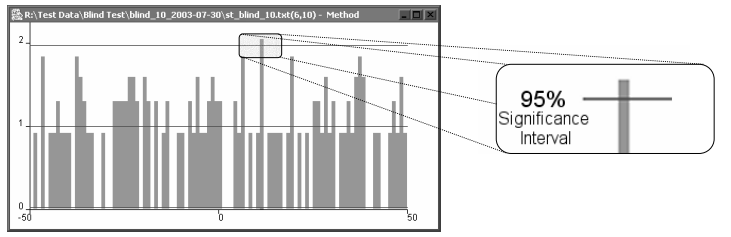

Figure 4-25 The cross-correlogram of spike trains 6 and 10 of trail three. Note that there are no truly signification peaks in this cross-correlogram

Closer inspection of the cross-correlograms, for these pairs, shows a peak barely higher than the confidence interval, see Figure 4-25. For this reason, these correlations can be discounted. Thus, it is possible to conclude that two independent groups exist.

\subsubsection{The upper group of trial three}

In order to interpret the upper group of trial three, the top portion of the correlation grid is enlarged, as shown in Figure 4-26.

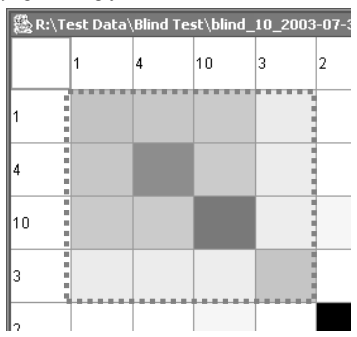

Figure 4-26 Enlargement of the upper group of the correlation grid of trial three data

Note that the upper group consists of neurons 1, 4, 10 and 3 , as ordered in the grid. Further, there are strong correlations between spike trains 1,4 and 10 . Also note that spike train 3 correlates, relatively weakly, with spike trains 1, 4 and 10 . Further to closer inspection of their cross-correlograms, it is possible to deduce that neuron 1 connects to neurons 3, 4 and 10, as shown in Figure 4-27.

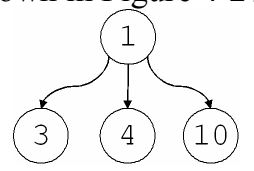

Figure 4-27 The neuronal assembly of the upper group of trial three

In addition, it is possible to observe that the strength of the connection between neurons 1 and 3, see Figure 4-28 for the cross-correlogram, is weaker than that of the connections between neurons pairs $(1,4)$ and $(1,10)$, see Figure 4-29. 


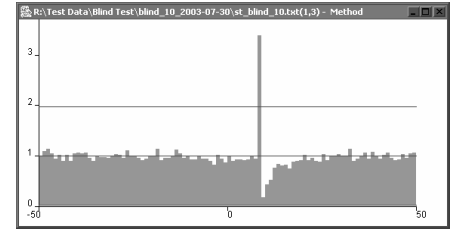

Figure 4-28 The cross-correlogram of spike trains 1 and 3 of trail three

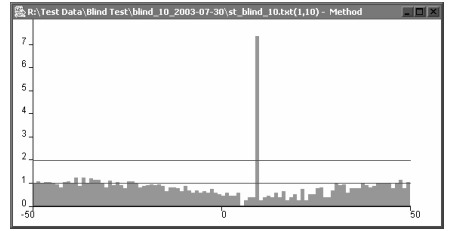

Figure 4-29 The cross-correlogram of spike trains 1 and 10 of trail three

\subsubsection{The lower group of trial three}

In order to interpret the lower group of trial three, the lower portion of the correlation grid is enlarged and is shown in Figure 4-30.

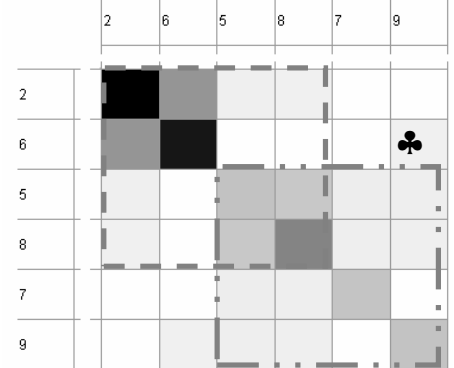

Figure 4-30 Enlargement of the lower group of the correlation grid of trial three data

From this figure, two main, overlapping groups are apparent. The top group consists of neurons 2, 6, 5 and 8; and the bottom group which consists of neurons 5, 8, 7 and 9. Recall from section 4.1.4, that overlapping groups tend to indicate different, connected hierarchies.

The interpretation of the bottom group is relatively straight-forward, it has a similar pattern and structure to the upper group of this trial. The bottom group depicts neuron 5, one level higher than neurons 7,8 and 9 .

The top group depicts neuron 2 connecting to both neurons 5 and 6 . In addition, a connection exists from neuron 2 to neuron 8 . It is likely that this is attributable to the connection from neuron 1 to neuron 8 via neuron 5. By examining the details of the cross-correlogram (not shown due to space limitations), between spike trains 2 and 8 , it is possible to verify this hypothesis. The link between the top and bottom groups is clearly via neuron 5 . In addition to these relationships, the grid shows a second link between the two groups, from neuron 6 to neuron 9, indicated in Figure 4-30 by the following symbol $(\boldsymbol{*})$.

\subsubsection{Summary of trial three observations}

From this analysis, the overview and details of the correlation grid, it is possible to deduce the underlying neuronal assembly of the data set, as depicted in Figure 4-31.

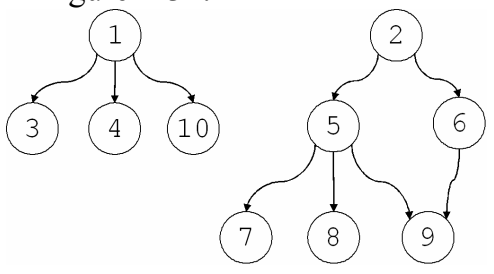

Figure 4-31 The neuronal assembly of trial three

\section{Conclusions}

This correlation grid, which is based on information visualisation, has proven to be an effective tool in supporting the study of synchronous spiking in multi-dimensional neuronal systems. This method has helped us to define the unknown structure of connections between neurons. At this stage, only a small number of simulated data sets have been analysed. However, these initial empirical studies have yielded successive correct connection architectures.

These empirical studies are continuing with a variety of datasets from larger and more diverse assemblies. The results from these studies will be published in the future.

\section{Future Work}

The Correlation Grid will be developed to support software functionality such as (i) greater direct user manipulation, (ii) the facility to "zoom" and "hide" data in the grid, (iii) the capability to vary the thresholds of "significance" which underlie the colour coding, (iv) encoding other information such as the number of peaks \& peak delay in each individual cross correlogram and (v) multiple threshold colour coding will be introduced.

\section{Acknowledgements}

This research is supported by the Engineering and Physical Sciences Research Council (EPSRC) grant number GR/N04904. Additionally, the research of Roman Borisyuk is supported in part by the Russian Foundation of Basic Research (grant 03-04-48482).

\section{References}

Awiszus F., 1997. Spike train analysis. Journal of Neuroscience Methods, 74.

Borisyuk R. M., 2002. Oscillatory activity in the neural network of spiking elements. BioSystems, 67:3-16

Brillinger D. R., 1979. Confidence intervals for the crosscovariance function. Selecta Statistica Canadiana, V:1-16. 
Gerstein G. L., Kirkland K. L., 2001. Neural assemblies: technical issues, analysis, and modelling. Neural Networks, 14(6-7):569-598

MuLab http://mulab.physiol.upenn.edu/index.html

Nase G., Singer W., Monyer H., Engel A. K., 2003. Features of neuronal synchrony in mouse visual cortex. Journal of Neurophysiology, August 90(2):1115-1123

Neuenschwander S., Castelo-Branco M., Baron J., Singer W., 2002. Feed-forward synchronization: propagation of temporal patterns along the retinothalamocortical pathway. Philos Trans R Soc Land B Boil Sci December, 29;357(1428):1869-1876

Schmidt M. F., 2003. Pattern of interhemispheric synchronization in $\mathrm{HVc}$ during singing correlates with key transitions in the song pattern. Journal of Neurophysiology, August 27

Walter M., Stuart L., Borisyuk R., 2003. Spike Train Correlation Visualization. Proceedings of the 7 th IEEE International Conference on Information Visualization (IV03):555-560 